\title{
Elements of the great Comet of 1882 .
}

(Communicated by Vice-Admiral Rozean, Superintendent, U. S. Naval Observatory).

The following elements were computed from three observations made at the U.S. Naval Observatory; the first and last being made with the Transit circle, and the middle one compared with a known star which was after. wards observed on the Transit Circle:

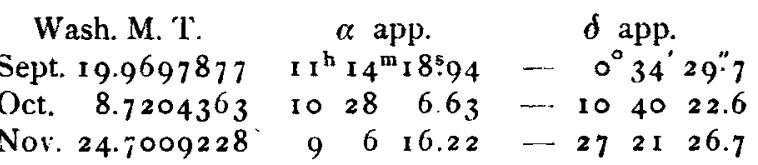

From these observations we deduce:

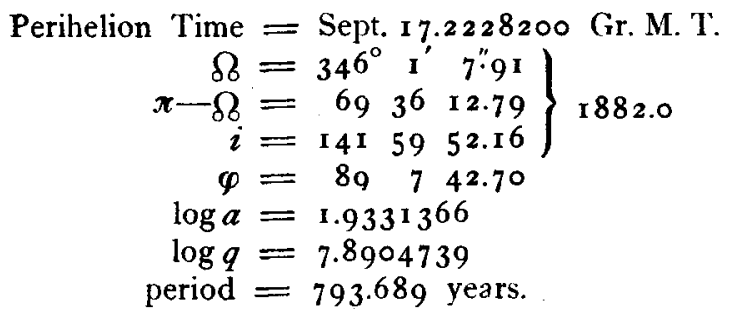

$$
\begin{aligned}
& \delta \lambda \cos \beta=-0.06 \\
& \delta \beta=+0.01 \text {. } \\
& x=r[9.9951411] \sin \left(170^{\circ} 42^{\prime} \text { I 2. }_{72}+v\right) \\
& y=r[9.9877234] \sin \left(262 \quad 4^{6} \quad 57.39+r\right) \\
& z=r[9.4435130] \sin (492025.11+v)
\end{aligned}
$$

The observations as given were afterwards corrected for parallax by means of elements previously computed. These elements bear a considerable resemblance to Comet I, B. C. 37 I and it may possibly be its third return, a very brilliant comet having been seen in full daylight A. D. 363 .

Washington, 1882 Dec. r 9 .

E. Frisby, Prof. Math., U.S. N.

\section{Der Venus-Durehgang 1882 Dec. 6.}

Von Prof. F. A. C. Oudemans, Director der Sternwarte in Utrecht.

Im Allgemeinen ist hier in den Niederlanden in diesem Monat die Iuft fast fortwährend bezogen gewesen, sehr oft haben wir sogar dicken Nebel gehabt. Am 6. December war es Morgens so schlecht wie möglich, doch hat sich etwa gleichzeitig mit dem Eintritt $\left(2^{\mathrm{h}} 2 \mathrm{o}^{\mathrm{m}}\right.$ M. Z. $)$ die Luft hier und dort aufgeklärt.

In Deventer und Enschede ist der Eintritt vom I. bis zum 2. Contact beobachtet, am letzten Orte von Dr. A. J. van Deinse, Director der Industrieschule daselbst, in der astronomischen Welt durch seine Doctor-Dissertation über den Cometen I $846 \mathrm{IV}$ bekannt, (welcher, beiläufig gesagt, nach van Deinse's Elementen am vergangenen 20. November sein Aphelium passirt hat.)

Zu Utrecht ist die Sonne nur während einiger Minuten sichtbar gewesen, und zwar mitten zwischen dem 1. und 2 .
Contact; hiervon verlor ich noch einen Theil, weil die Sonne sich hinter dem Windzeigermast des met. Instituts in unmittelbarer Nähe der Sternwarte verbarg; als sie wieder zum Vorschein trat, war Venus zu zwei Dritteln eingetreten, und war, eben wie beim Ausgang 1874 auf Réunion, der ganze Rand des Planeten ausserhalb der Sonne durch die beleuchtete Atmosphäre sichtbar. Herrn Dr. van Deinse fiel es auf, dass die leuchtende Venusatmosphäre im umkehrenden Fernrohr oben viel heller war als unten. Vielleicht war dies etwas früher, als ich die Sonne wiedersah; mir erschien der Bogen, (der etwa $120^{\circ}$ betragen haben wird), gleichmässig erleuchtet.

In Leyden war, wie College Bakhuyzen mir schrieb, die Luft ganz bezogen.

Utrecht 1882 Dec. 24.

7. A. C. Oudimans.

\section{In balt:}

Zu Nr. 2482. And. Lindstedt. Ueber die Integration einer gewissen Differentialgleichung. 145. - Freyvid Rancken. Ueber die Eigenbewegungen der Fixsterne. 149. - H. Kreutz. Elemente des grossen September-Cometen I882. I 57. - E. Frisby. Elements of the great Comet of 1882. 159. - F. A. C. Oudemans. Der Venus-Durchgang 1882 Dec 6.159. 